\title{
A Mechanical, Three-Dimensional, Ultrasound-Guided Breast Biopsy Apparatus
}

\author{
Kathleen JM Surry ${ }^{1}$, Wendy L Smith ${ }^{1,2}$, Gregory R Mills ${ }^{1}$, Donal B Downey ${ }^{1,3}$, \\ Aaron Fenster ${ }^{1,2,3}$ \\ ${ }^{1}$ Imaging Research, Robarts Research Institute, London Canada \\ ${ }^{2}$ Medical Biophysics, University of Western Ontario, London Canada \\ ${ }^{3}$ Radiology, London Health Sciences Centre, London Canada \\ kath@irus.rri.on.ca
}

\begin{abstract}
We have designed a prototype three-dimensional ultrasound (US) guidance apparatus to improve breast biopsy outcomes. Features from stereotactic mammography and free-hand US guided biopsy have been combined with 3D US imaging. This breast biopsy apparatus (BBA) accurately guides a needle into position for firing into target tissue. We have evaluated the BBA in three stages. First, by testing the placement accuracy of a needle in a tissue mimic. Second, with tissue mimic phantoms that had embedded lesions for biopsy. Finally, by comparison to free-hand US-guided biopsy, using chicken breast phantoms. The first two stages of evaluation quantified the mechanical biases in the BBA. Compensating for these, the BBA achieved a $96 \%$ success rate in targeting $3.2 \mathrm{~mm}$ 'lesions' in chicken breast phantoms. The expert radiologists performing biopsies with free-hand US guidance achieved a $94 \%$ success rate. This has proven an equivalence between our apparatus and free-hand biopsy, for $3.2 \mathrm{~mm}$ lesions in vitro, with a $95 \%$ confidence.
\end{abstract}

\section{Introduction}

\subsection{Breast Biopsy Apparatus (BBA) Development}

We have developed a prototype three-dimensional ultrasound (US)-guided breast biopsy apparatus (BBA) to improve patient outcomes (Figure 1). It is based on features from stereotactic mammography and free-hand ultrasound guided biopsy [13]. With this apparatus, we avoid the ionizing radiation associated with mammography, but retain the safe needle trajectory, parallel to the chest wall. Also, the real-time imaging associated with US is exploited, with the extra advantage of near real-time 3D US imaging.

A common problem with needle guidance systems for both stereotactic mammography and for the emerging MR breast imaging techniques is the lack of real-time information [4,5]. There are some groups reporting success for needle guidance in interoperative MRI units [6], but these systems are both rare and expensive. A fully robotic breast biopsy system is currently under development for 
use within a $1.5 \mathrm{~T}$ whole body magnet [7], which shows promise, but still requires an expensive and non-portable MRI unit.

The apparatus shown in Figure 1 is a prototype built specifically for testing our biopsy concept with phantoms. The degrees of freedom, apart from the rotational mover for the 3D US scanning, are all manually adjustable. The needle guide (A) and the US probe (B) are kept in the same line by the stage at C (moves in the $\mathrm{Z}$ direction). The needle guide may be moved off axis in $\mathrm{z}$ (at D) to target a location seen in US at some angle other than zero degrees (straight down). The needle guide is also moved vertically (E, in the y-direction) to target at any depth in the volume. The US probe, in this case a transrectal, side-firing $7 \mathrm{MHz}$ model, is rotated on its axis by the mover at $\mathrm{F}$ to collect a $3 \mathrm{D}$ volume. It can be moved, on-axis with the needle (the $\mathrm{x}$-direction), by the fourth stage at G. Needle insertion depth, in the same direction, is indicated by a ruler attached to the needle guide.

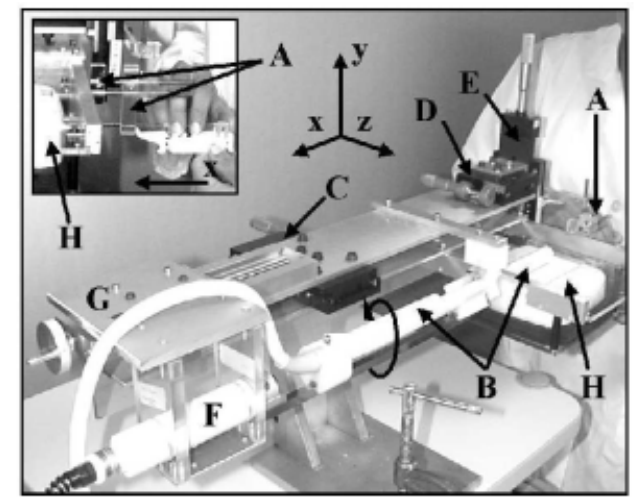

Fig. 1. The prototype three-dimensional ultrasound guided breast biopsy apparatus (BBA), with needle guide shown in inset. A Needle guide. B US probe. C, D, E, G Translational movers. F Rotational mover. $\mathbf{H}$ Compression plate. The system co-ordinates are also indicated as (x, y, z).

2D US images from an Acuson 128 ultrasound machine were digitised and shown as a 3D image within one second by a Power Macintosh 7500 [8]. 3D multi-planar reformatting tools were used to view the $3 \mathrm{D}$ volume in any orientation, and to identify a biopsy target point [9-11]. These co-ordinates were used in transformation equations to provide the necessary adjustments to line up the US probe and needle guide with the target. The needle was inserted and positioned under 2D US guidance and the needle gun was fired to acquire a sample of tissue. At any time during the procedure, 3D US images may be acquired to check positioning. A post-biopsy image was often useful, allowing for confirmation that the needle was penetrating the volume.

\subsection{BBA Evaluation}

We evaluated this apparatus in three stages. First, we determined its accuracy in needle placement at a specified target point in three dimensions. Then, to include the firing of the biopsy gun and the acquisition of a sample, we tested the prototype's 
biopsy success rates in targeting 'lesions' of known size, between $1.6 \mathrm{~mm}$ and $15.9 \mathrm{~mm}$ in diameter. Finally, we designed an experiment which compared the biopsy success rates of our 3D US guided BBA with expert radiologists performing freehand US guided biopsy. In this manner, we have been able to evaluate our prototype BBA so that it may be rebuilt for clinical testing.

\section{Methods}

\subsection{Needle Placement Accuracy}

A total of $720.79 \mathrm{~mm}$ (1/32 inch) beads were placed in agar blocks [12], with a line at each of the four combinations of needle penetration depth $(3.5 \pm 0.5 \mathrm{~mm}$ and $17.5 \pm 0.5 \mathrm{~mm})$ and vertical depth $(12 \pm 0.5 \mathrm{~mm}$ and $28 \pm 0.5 \mathrm{~mm})$ (Figure 2). These blocks were fixed to the BBA under the top compression plate at $\mathrm{H}$. A target was found using 3D US and the apparatus (both the probe and the needle guide) was moved into position. Another 3D US confirmed the bead's position, and a 14 gauge needle was inserted to these co-ordinates, without 2D US guidance. Another 3D volume was acquired with the needle in place.

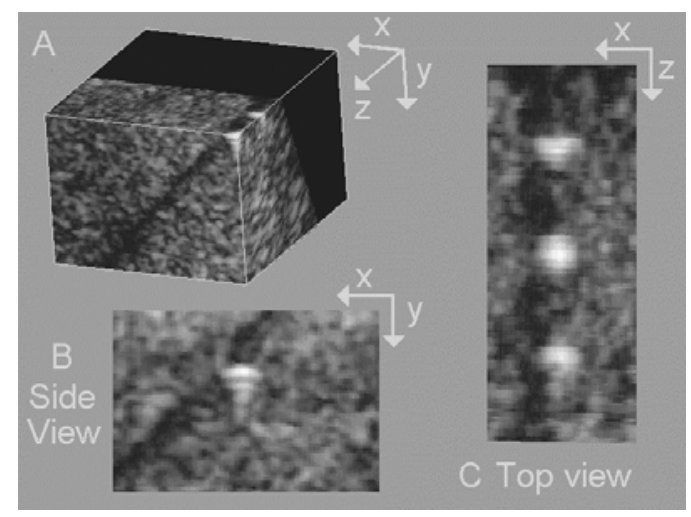

Fig. 2. Small, $0.79 \mathrm{~mm}$ beads in an agar phantom, shown here in three-dimensional ultrasound.

Three observers identified the bead's position in the pre-insertion US images, and the needle tip's position in the post-insertion US images. These measurements were made three times by each observer. Needle tip placement relative to bead position was plotted in three dimensions for all combinations of penetration depth and vertical depth. Using 3D principal components analysis (PCA) [13], the maximum variance was found in the needle's placement from the target bead. 


\subsection{Biopsy Accuracy}

To evaluate the biopsy accuracy of the BBA, poly(vinyl alcohol) cryogel (PVA-C) was used as a tissue mimic, due to its robust mechanical properties [14]. Green coloured PVA-C cylinders were embedded as 'lesions' into blocks of white PVA-C (Figure 3). The simulated lesions had equal length and diameter dimensions and were all placed with their centres at the same penetration distance and depth in the US image. The phantoms containing the $1.6 \mathrm{~mm}, 3.2 \mathrm{~mm}$ and $4.8 \mathrm{~mm}$ diameter cylinders held 24 samples of each. The phantom with $6.4 \mathrm{~mm}$ diameter lesions held 21 samples, the $9.5 \mathrm{~mm}$ lesion phantom held 18 and the $15.9 \mathrm{~mm}$ lesion phantom held 12. Every lesion was biopsied once only.

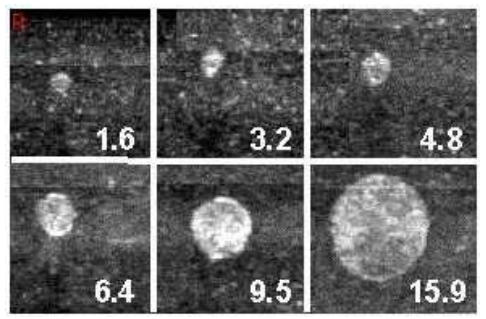

Fig. 3. PVA-C lesions in PVA-C tissue, shown in ultrasound. The lesions' diameters are given in $\mathrm{mm}$.

Similarly to the needle placement study, a 3D US image was acquired and the target identified. The co-ordinates for the centre of the target lesion were used in the transformation equations and the needle inserted. The needle's position was not updated with 2D US guidance. The needle was then fired, using the Bard Magnum biopsy gun (Bard, GA 30014). The biopsy needle acquires a sample by first firing forward a notched central annulus, then a covering sheath which slices off a section of tissue into the notch. This notch is $19 \mathrm{~mm}$ long, giving some margin of error in this dimension. A post-biopsy 3D US image was acquired before the needle was removed. The biopsy sample was inspected for green PVA-C and a hit or miss was recorded.

The placement of the needle, relative to the target lesion's centre, was determined using the pre- and post-biopsy $3 \mathrm{D}$ images. A $2 \mathrm{D}$ PCA analysis was then performed to identify any placement bias in the needle.

\subsection{Comparison to Free-Hand US Guided Biopsy}

In order to establish whether the BBA is a suitable substitute for free-hand US guided biopsy, we compared the performance of the BBA to that of expert radiologists, in a 'realistic' biopsy task. Chicken breasts were implanted with $3.2 \mathrm{~mm}$ diameter PVA-C lesions (Figure 4). Three chicken breasts were sewn together for the radiologists, with 36 lesions in each 3-breast phantom (Figure 5). Each of the two radiologists had to target 55 lesions. Only those lesions which were obvious were targeted (Figure 4). Five smaller phantoms with only a single breast and 15 lesions implanted were made for the BBA testing, as our prototype has a constrained targeting volume (Figure 6). 
We also performed 55 biopsies using the BBA. The choice of sample sizes was based on a two group test of equivalence in proportions with large unequal n's [15]. The one-sided test significance level is $10 \%$, and the power was $76 \%$, with a predicted success rate of $90 \%$ for both the radiologists and the BBA. This required a sample size of 55 for the test subject (BBA) and 110 samples for the standard for comparison (two radiologists).

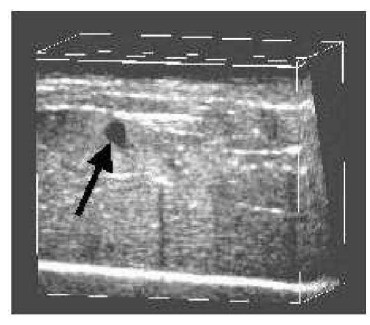

Fig. 4. A 3.2 mm PVA-C lesion in a chicken breast phantom, shown in 3D US.

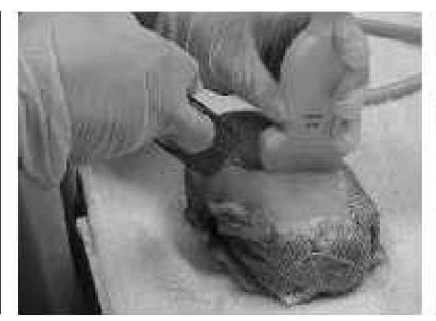

Fig. 5. Free hand US guided biopsy performed in a chicken phantom.

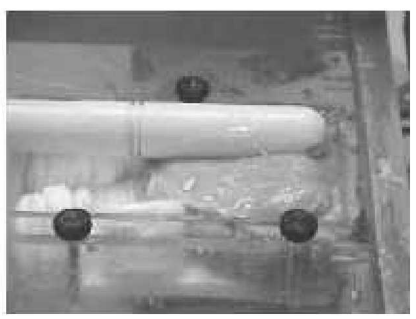

Fig. 6. BBA biopsy set-up with a chicken phantom.

\section{Results}

\subsection{Needle Placement Accuracy}

The largest inter- and intra-observer standard errors of measurement in this study were found for the bead measurement, in the z-direction of our system (see Figure 1), which has the poorest resolution in the reconstructed US image.

The 3D PCA analysis identified that the largest variance $(0.73 \mathrm{~mm})$ is found for the deepest beads at the shortest penetration distance. The smallest variance, $0.42 \mathrm{~mm}$, occurs for the shallowest beads, also at short penetration. The axes of maximum variance for each position was not along any of the co-ordinate axes of the machine. Variances along the secondary and tertiary PC axes ranged from 0.31 to $0.13 \mathrm{~mm}$ and 0.24 to $0.05 \mathrm{~mm}$, respectively. The largest bias in needle placement was for the deepest beads, at the shortest penetration distance, and was $1.16 \mathrm{~mm}$. The smallest bias was $0.69 \mathrm{~mm}$ for the shallowest bead and shortest penetration distance. Also, we can target to within $0.85 \mathrm{~mm}$ in the $\mathrm{x}-\mathrm{y}$ plane, with a $95 \%$ confidence.

\subsection{Biopsy Accuracy}

The post-biopsy 3D US images allowed for identification of the needle's final position. This clearly showed a bias in the placement of the needle's centre line, $0.14 \mathrm{~mm}$ too high, and $0.51 \mathrm{~mm}$ to the right of centre (Figure 7). No lesions were missed due to misplacement in the needle's axis direction, due to the length of the sampling notch. It was also identified that the needle's sampling notch provided a 
vertical bias to the tissue sampled. The bevel at the needle's tip directs the tissue at this level down and under the $2 \mathrm{~mm}$ diameter shaft and it is this tissue that is sampled by the sampling notch (Figure 8). Since the targeting was done with the central axis of the needle, rather than the bevel tip edge, this contributed to a sampling off-set of 1 $\mathrm{mm}$, and may have affected the results in biopsying the smaller lesions. The biopsy rates are shown in Figure 9. The total variance described by this test was $1.72 \mathrm{~mm}$ (standard deviation of $1.31 \mathrm{~mm}$ ).

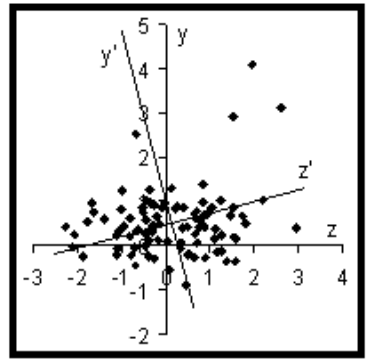

Fig. 7. Scatter plot of needle placement relative to lesion centre $(0,0)$. PCA axes $\left(z^{\prime}, y^{\prime}\right)$ are shown at the bias centre.

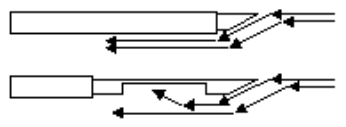

Fig. 8. Tissue at the needle's tip is directed down under the needle and is sampled by the sampling notch.

Using the minimum and maximum standard deviations along the PC axes, and including the bias caused by the bevel and sampling notch, we developed a model to fit the biopsy values (Figure 9). Using these results, the number of passes needed for a successful biopsy of any lesion size, at a given confidence level can be predicted. The number of passes for $95 \%$ confidence in hitting a $3 \mathrm{~mm}$ lesion is 5 , for example. Six passes are typically used and are well tolerated by patients [2].

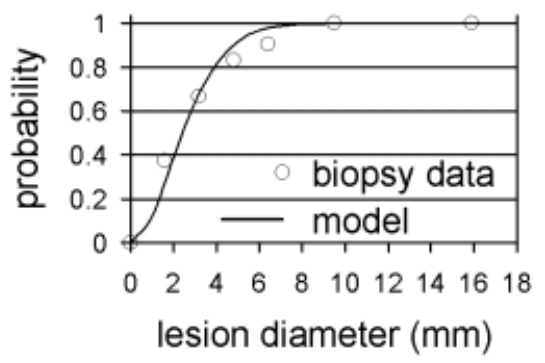

Fig. 9. Biopsy data and the model derived from the PC analysis and the needle notch sampling bias.

\subsection{Comparison to Free-Hand US Guided Biopsy}

With improvements made based on the first two evaluation experiments, we were able to achieve a $96 \%$ success rate with the BBA for $3.2 \mathrm{~mm}$ diameter lesions. By a 
comparison to the expert radiologists, who had a $94 \%$ success rate, we have shown our hypothesis, which was that the BBA is equivalent to free-hand biopsy, for this in vitro study. The one-sided $95 \%$ confidence interval demonstrates that the two biopsy methods are equivalent, within $5 \%$.

The use of chicken phantoms for testing breast biopsy procedures was successful, by providing clearly visible lesions in a tissue-like setting, both when viewed using US and upon biopsy.

\section{Discussion}

Since our experiments focused on biopsy accuracy and not on detection of 'lesions', our studies avoided any problems of target identification in ultrasound imaging. The targets were always obvious, and, if not, as was sometimes the case in the inhomogeneous chicken breasts, then that particular target was not attempted. This avoided a positive bias for the expert radiologists participating in the study.

We have shown, in three stages, that our breast biopsy apparatus performed well. Through an in vitro study, we have shown that this approach is a suitable alternative to free-hand US guided biopsy procedures for $3.2 \mathrm{~mm}$ lesions.

By performing the bead targeting study, we were able to establish the targeting accuracy of the BBA's mechanical stages and needle guide under ideal conditions. With the addition of the biopsy part of the procedure, the additional biases were modelled.

It was clear that the 3D US images were important in evaluating the procedure, as they provided information about a placement bias. This was evident in the PVA-C biopsy study, which could have been interpreted without knowledge of the bias, and the low targeting rate attributed solely to a larger scatter in a random normal distribution about the target point. Knowledge of the bias allowed us to improve our targeting success rate from $67 \%$ to $96 \%$ at $3.2 \mathrm{~mm}$.

Using artificial 'lesions' of known geometry and size have allowed us to compare and rate biopsy procedures. The strictly controlled PVA-C biopsy study allowed us to identify a clear bias in our procedure and assign its origin to the mechanical components, not to any inhomogeneities or other difficulties in the phantom. The chicken breast study provided a comparison of our new procedure to a clinical standard in a more realistic way. We have shown that the BBA provides an equivalent success rate to expert free-hand US guided biopsy. With the additions of 3D imaging, real-time needle insertion monitoring, and safety features which keep the needle away from the patient's chest wall, this BBA could eventually be used in a clinical setting and achieve results equal to an expert using free-hand US guided biopsy.

Improvements must be made to the apparatus before clinical testing. Besides the obvious cosmetic and ergonomic adjustments, we are also automating the movers to increase the speed of the procedure. When a target is identified by the clinician in the $3 \mathrm{D}$ US image, the machine will move the US probe and the needle guide into position. We will also be increasing the available target volume, which was suitable for our phantom studies, but not for a clinical setting. 


\section{References and Acknowledgements}

We thank S Odegaard for helping with the US data collection and Dr A Kumar for performing the chicken biopsies. Also, for Dr M Eliasziw's help with the chicken breast statistics. This work was funded by the Canadian Institutes of Health Research (CIHR) and London Health Sciences Centre (LHSC).

1 Roe SM, Mathews JW, Burns RP, Sumida MP, Craft P and Greer MS 1997 Stereotactic and ultrasound core needle breast biopsy performed by surgeons Am J Surg 174 699-704

2 Liberman L, Feng TL, Dershaw DD, Morris EA and Abramson AF 1998 US-guided core breast biopsy: Use and cost-effectiveness Radiology 208 717-723

3 Meyer JE, Smith DN, Lester SC, et al 1999 Large-core needle biopsy of nonpalpable breast lesions JAMA 281 1638-1641

4 Dershaw DD 2000 Equipment, technique, quality assurance, and accreditation for imagingguided breast biopsy procedures Radiol Clin North Am 38(4) 773-789

5 Heywang-Köbrunner SH, Heinig A, Schaumlöffel U, et al 1999 MR-guided percutaneous excisional and incisional biopsy of breast lesions Eur Radiol 9 1656-1665

6 Daniel BL, Birdwell RL, Ikeda DM, et al 1998 Breast lesion localization: A freehand, interactive MR imaging-guided technique Radiology 207 455-463

7 Kaiser WA, Fischer H, Vagner J, Selig M 2000 Robotic system for biopsy and therapy of breast lesions in a high-field whole-body magnetic resonance tomography unit Invest Radiol 35(8) 513-519

8 Tong S, Downey DB, Cardinal HN and Fenster A 1996 A 3D ultrasound prostate imaging system Ultrasound Med Biol 22 735-746

9 Nelson TR, Downey DB, Pretorius DH and Fenster A 1999 Three-dimensional ultrasound. Lippincott Williams \& Wilkins, Philadelphia

10 Fenster A and Downey DB 2000 Three-dimensional ultrasound imaging In: Annual Review Biomed Eng 2000: Annual Reviews 457-475

11 Fenster A and Downey DB 1996 3D ultrasound imaging: A review 1996 IEEE Eng Med Biol 15 41-51

12 Rickey DW, Picot PA, Christopher DC and Fenster A 1999 Three-dimensional ultrasound. Lippincott Williams \& Wilkins, Philadelphia

13 Drury SA 1993 Image Interpretation in Geology $2^{\text {nd }}$ edition. Chapman and Hall

14 Chu KC and Rutt BK 1997 Polyvinyl alcohol cryogel: An ideal phantom material for MR studies of arterial flow and elasticity Mag Res Med 37 314-319

15 Farrington CP and Manning G 1990 Test statistics and sample size formulae for comparative binomial trials with null hypotheses of non-unity relative risk Stat Med 9(12) 1447-1454 